\title{
New Evidence of Chromospheric Evaporation
}

\author{
J. Kašparová ${ }^{1}$, P. Kotrč and P. Heinzel \\ Astronomical Institute of the Academy of Sciences of Czech Republic, \\ CZ-251 65 Ondrejov, Czech Republic
}

I. F. Nikulin

Sternberg Astronomical Institute, RU-119899 Moscow, Russia

P. Rudawy

Astronomical Institute of the Wroclaw University, PL-51 622 Wroclaw, Poland

\begin{abstract}
H} \alpha$ spectra obtained at Sternberg Astronomical Institute in Moscow during the gradual phase of the flare on May 16, 1981 are analysed. The flare-ribbon profiles show a red asymmetry of the emission peaks, and we interpret this as an absorption in the blue wing of the profile due to expected upflows. We demonstrate a good correlation between the observed $\mathrm{H} \alpha$ profiles and those computed with the flare model F1 and upflows reaching $10 \mathrm{~km} \mathrm{~s}^{-1}$.
\end{abstract}

\section{Introduction}

During the impulsive phase of solar flares, an explosive evaporation scenario is commonly accepted. Fast upflows in the transition region and simultaneous downflows in the cooler chromospheric part are usually observed by X-ray spectrometers (SMM, Yohkoh) and by optical spectrographs, respectively. In the case of downflows, a blue asymmetry can be detected, e.g., in the hydrogen $\mathrm{H} \alpha$ line, which was recently explained by Heinzel et al. (1994). On the other hand, the plasma flows are much slower during the gradual phase of a typical two-ribbon flare and, as found by Schmieder et al. (1987), also cool chromospheric plasma exhibits slow upflows, named a 'gentle evaporation', in contrast to the explosive one. This gentle evaporation as deduced from the $\mathrm{H} \alpha$ line was evidenced by Schmieder et al. (1987) using the MSDP observations of large two-ribbon flares. In the flare ribbon, the blue-shifted absorption profile is consistent with upward chromospheric flows of $0.5-10 \mathrm{~km} \mathrm{~s}^{-1}$. One of the observed flares was the event of May 16, 1981 for which a series of spectra in $\mathrm{H} \alpha$ and CaII lines was also recorded at Sternberg Astronomical Institute in Moscow. In this paper we analyse these $\mathrm{H} \alpha$ spectra and demonstrate that in some places rather faint emission peaks appear in the ribbons, which is consistent with the

\footnotetext{
${ }^{1}$ Astronomical Observatory Valašské Meziřící, CZ-757 01 Valašské Meziříćí, Czech Republic
} 
F1 flare model of Machado et el. (1980) but was not observed by MSDP. The observed red asymmetry of these emission peaks is then interpreted as a natural consequence of gentle-evaporation upflows in flare ribbons. Note that post-flare loops pertinent to this event have been extensively studied by Schmieder et al. (1987), Heinzel et al. (1992) and, recently, by Gu et al. (1997).
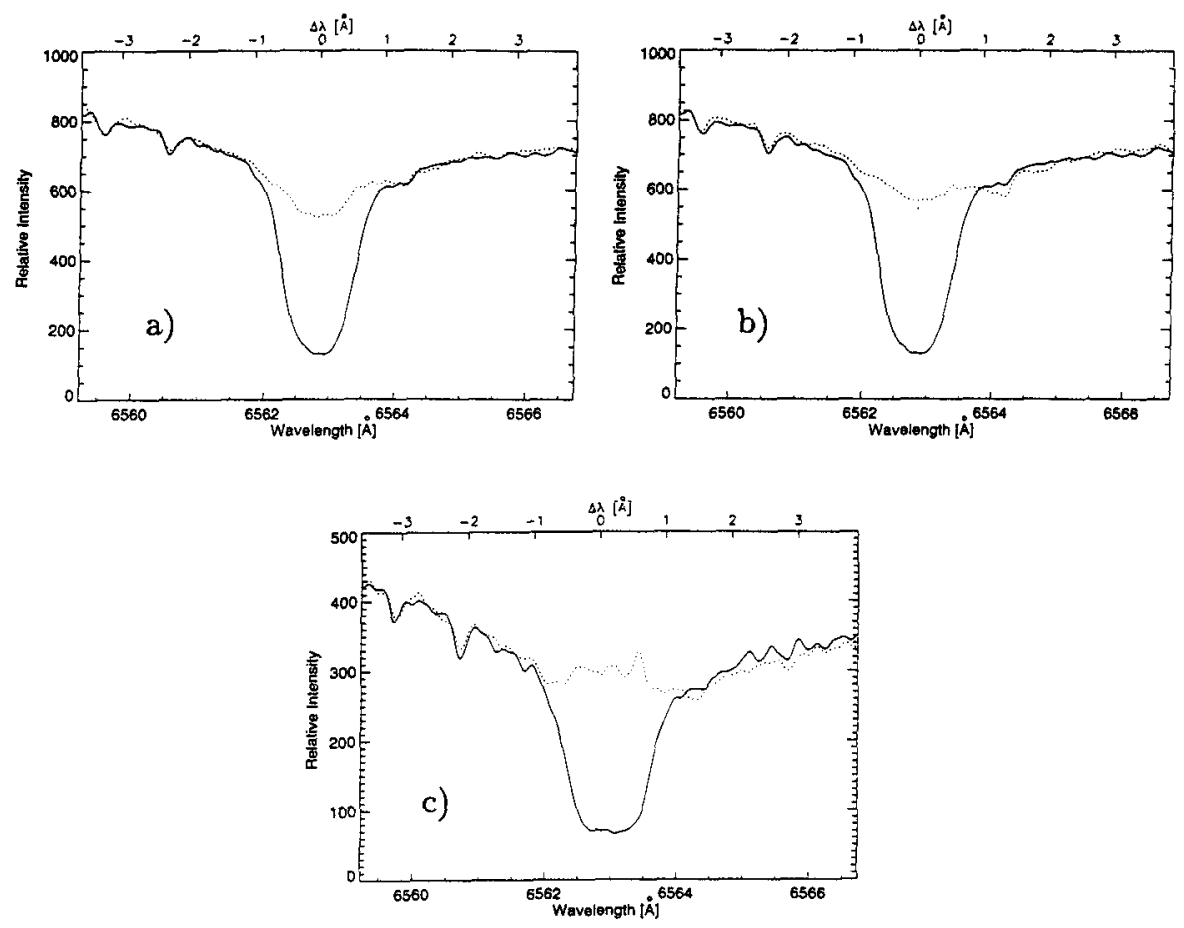

Figure 1. Profiles of the $\mathrm{H} \alpha$ flare ribbon with the enhanced emission (dashed line); solid line represents the adjacent quiet-chromosphere line profile. $\mathbf{a}-09: 08 \mathrm{UT}, \mathbf{b}-09: 08 \mathrm{UT}$ (closer to the center of the loops), c - 09:26 UT.

\section{Observations}

This 3B/X1 flare (in NOAA active region 3106 on May 16, 1981 at $12 \mathrm{~N} 10 \mathrm{E}$ ) started at 08:14 UT after (07:54 UT) eruption of the filament (Nikulin et al. 1986, Schmieder et al. 1987). The $\mathrm{H} \alpha$ photographic spectra were obtained at the solar tower telescope of the Sternberg Astronomical Institute in Moscow between 09:08 UT and 10:15 UT. As the maximum of the flare was reported at 08:54 UT ( $\mathrm{Gu}$ et al. 1997), the spectra were taken during the gradual phase of the flare with well developed post-flare loops and flare ribbons. The spectrograph slit was placed on the bright ribbon above which the dark post-flare loops also occurred. Details concerning the telescope and the flare observation were described by Nikulin et al. (1986). The linear dispersion in $\mathrm{H} \alpha$ was $0.7 \AA \mathrm{mm}^{-1}$. Spectra were digitized using the Wroclaw Observatory microphotometer. 
$\mathrm{H} \alpha$ profiles in the flare ribbon are strongly enhanced as compared to the quiet-Sun profiles - see Figure 1 for three examples. The profile in Figure 1a resembles the MSDP profile displayed in Figure 5 of Schmieder et al. (1987), with a blue-shifted line core and an enhanced emission in the red wing. However, in all our examples we see a small emission peak in the red wing, which is very prominent in Figure 1c. There is also an indication of an emission peak in the blue wing, namely in Figure 1c. Absorption clearly visible in the far red wing (Figures $1 b, c$ ) is consistent with large downflows in cool post-flare loops which probably partly overlay the ribbon (see also Figure 5 in Schmieder et al. 1987). The fact that MSDP observations do not indicate small peaks similar to those visible in our Figures 1b,c may be due to the lower spectral resolution, around $0.3 \AA$ or an order of magnitude lower than in our case.

\section{Interpretation of the Red Asymmetry}

Since the enhanced ribbon emission is roughly similar to that produced by a standard flare model, F1 of Machado et al. (1980), we use this model to simulate numerically the emission peak asymmetry (the temperature structure of the $\mathrm{F} 1$ model is shown in Figure $2 \mathrm{~b}$ ). As already mentioned in the Introduc-
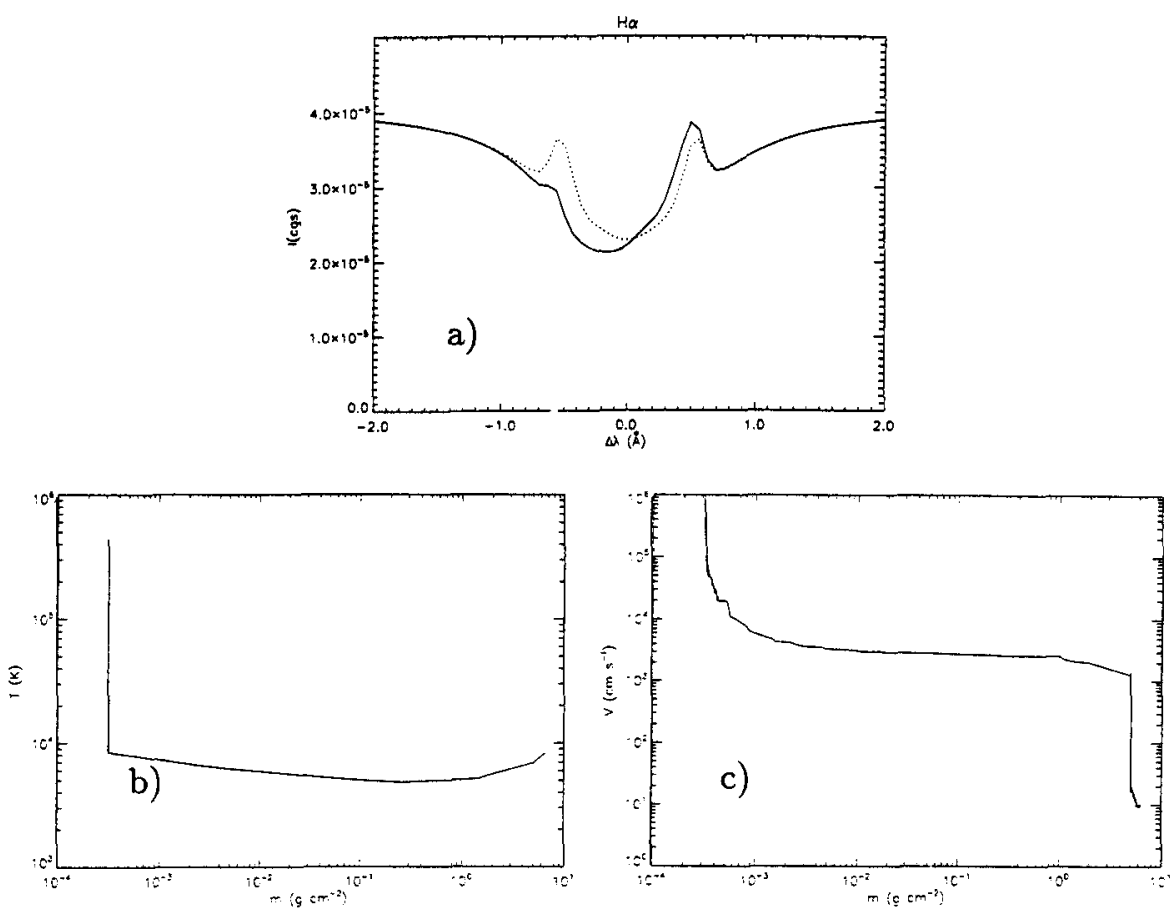

Figure 2. Results of numerical simulations: $\mathbf{a}$ - theoretical profiles, b - temperature structure of F1 model, $\mathbf{c}$ - velocity field. 
tion, Heinzel et al. (1994) have explained a similar, but blue asymmetry by chromospheric downflows imposed on the F1 model. By considering the reverse case of upflows consistent with the gentle evaporation, one would expect the red asymmetry with an enhanced red emission peak, but with the blue-shifted line core (self-absorption profile). This is what we actually observe in our spectra. Numerical simulations of $\mathrm{H} \alpha$ asymmetries in flares were done by Nejezchleba (1998), who also computed an example shown in Figure 2a. The dotted profile with two symmetrical emission peaks corresponds to the static model F1. If we impose an upflow of up to $10 \mathrm{~km} \mathrm{~s}^{-1}$ (Figure 2c) in the upper chromosphere, the red peak becomes more intense and, simultaneously, the line core shifts to blue. The actual effect depends on the velocity gradient, the location of the flow in the chromosphere and the flare model itself (Nejezchleba 1998 and private communication). We see that the blue peak is significantly lowered by an absorption within an upward moving plasma. In this flow, the absorption is lower in the red peak and, thus, we see more emission from deeper ribbon layers. Note that asymmetrical peaks are the result of an interplay between the height variations of the $\mathrm{H} \alpha$ source function and the velocity field.

\section{Conclusions}

Comparing line profiles obtained with good spectral resolution with results of numerical simulations, we interpret the red asymmetry of the enhanced $\mathrm{H} \alpha$ emission as a consequence of gentle-evaporation upflows in flare ribbons. This supports the previous conclusions of Schmieder et al. (1987).

Acknowledgments. This work was supported by the grant GA CR 1199 and the Key-project K1-003-601.

\section{References}

Gu, X.-M., Ding, Y.-J. and Schmieder, B. 1997 A\&A, 324, 289

Heinzel, P., Kotrč, P., Karlický, M. and Švestka, Z. 1994, Solar Phys., 152, 393

Heinzel, P., Schmeieder, B. and Mein, P. 1992, Solar Phys., 139, 82

Machado, M. E., Avrett, E. H., Vernazza, J. E. and Noes, R. W. 1980, ApJ, 242,336

Nejezchleba, T. 1998, A\&A, 127, 607

Nikulin, I. F., Alikaeva, K. V. and Polupan, P. M. 1986, Soln. Dannye, Byull. 1986, No. 1, 89 (in russian)

Schmieder, B., Forbes, T. G., Machado, M. E. and Malherbe, J. M. 1987, ApJ, 317,956 